\section{Perfil sociodemográfico e comorbidades psiquiátricas em dependentes químicos acompanhados em comunidades terapêuticas}

\author{
Sociodemographic profile and psychiatric comorbidities in chemical \\ dependent patients accompanied in therapeutic communities
}

Rafael Vinícius Danieli', Mírian Brusadelli Macedo Ferreira', Julia Melloni Nogueira', Leonardo Nunes de Castro Oliveira', Emirene Maria Trevisan Navarro da Cruz' ${ }^{1}$ Gerardo Maria de Araújo Filho'

\section{RESUMO}

Objetivos: Analisar o perfil sociodemográfico, antecedentes pessoais/familiares e a prevalência de comorbidades psiquiátricas em uma amostra de dependentes de álcool e/ou substâncias psicoativas (SPAs) acompanhados em duas comunidades terapêuticas. Métodos: Trata-se de um estudo de prevalência, com coleta de dados realizada em uma amostra de 90 entrevistados por meio da aplicação de uma ficha de dados sociodemográficos/antecedentes pessoais e familiares e do Mini International Neuropsychiatric Interview versão Plus (MINI Plus). Os entrevistados foram divididos em três grupos: etilistas (grupo A), dependentes de SPAs ilícitas (Grupo B) e dependentes de álcool e SPAs ilícitas (grupo C). Resultados: Observou-se prevalência elevada de comorbidades psiquiátricas $(88,8 \%)$ e, em específico, do transtorno depressivo maior (TDM) no grupo A $(36,7 \%)$ e do transtorno de personalidade antissocial nos grupos B e C (46,7\% e 30\%, respectivamente). Foi possível traçar perfis próprios para cada grupo, havendo convergência quanto aos dados sociodemográficos para os grupos A e C e entre os grupos B e C para as comorbidades psiquiátricas. Conclusão: A vulnerabilidade sociodemográfica e a alta prevalência de comorbidades psiquiátricas denotam a fragilidade da população de dependentes químicos. Os perfis próprios de cada grupo evidenciam a importância de tratamento individualizado.

\begin{abstract}
Objectives: Analyze the sociodemographic profile, personal/family antecedents and the prevalence of psychiatric comorbidities in a sample of alcohol and/or psychoactive substances (PAS) accompanied in two therapeutic communities. Methods: This is a prevalence study, having gathered data from a sample with 90 interviewee through a form with sociodemographic/personal/family antecedents data and the Mini International Neuropsychiatric Interview (MINI Plus). The interviewee were divided in three groups: alcohol dependent (group A), illicit PAS dependents (group B) and alcohol and illicit PAS (group C). Results: A high prevalence of psychiatric comorbidities (88.8\%) and, specifically, the major depressive disorder (MDD) in group A (36.7\%) and antisocial personality disorder in groups B and C
\end{abstract}

1 Faculdade de Medicina de São José do Rio Preto (Famerp) - Departamento de Psiquiatria.

Recebido em

$31 / 12 / 2016$

Aprovado em

$22 / 7 / 2017$

DOI: 10.1590/0047-2085000000163
Endereço para correspondência: Rafael Vinícius Danieli Faculdade de Medicina de São José do Rio Preto (Famerp) Jaborandi, SP, Brasil

E-mail: rafaeldanieli@ig.com.br 


\section{Keywords}

Chemical dependence, mental disorders, psychiatric comorbidities, psychoactive substances, therapeutic communities.
( $46.7 \%$ and $30 \%$, respectively) was found. It was possible to track characteristic profile for each group, having found a convergence of sociodemographic data for groups A and C, and between groups B and C, for psychiatric comorbidities. Conclusion: The sociodemographic vulnerability and the high prevalence of psychiatric comorbidities denote the fragility of the population of chemical dependents. The characteristic profiles for each group demonstrate the importance of an individualized treatment.

\section{INTRODUÇÃO}

A dependência do álcool e das substâncias psicoativas (SPAs) ilícitas representa um problema de saúde pública, dada sua prevalência elevada e os prejuízos socioeconômicos e pessoais ${ }^{1-3}$. No Brasil, as prevalências de dependência de álcool, maconha e estimulantes são de, respectivamente, 12,3\%, $1,2 \%$ e 0,2\% ${ }^{4}$. Considerando a população mundial em 2013, cerca de 27 milhões de pessoas tinham algum transtorno decorrente do uso de drogas ou dependência química ${ }^{5}$.

A dependência de álcool e SPAs acarreta redução da capacidade laboral, desemprego, marginalização/criminalidade, desestruturação familiar, aumento da demanda aos serviços de saúde e comorbidades psiquiátricas e clíni$\operatorname{cas}^{1,6-11}$. Acredita-se que conhecer o perfil sociodemográfico e as demandas dos dependentes químicos seja fundamental para uma melhor assistência, prevenção de casos novos/recaídas e tratamento dos já existentes ${ }^{12-16}$. Além disso, dada à alta associação da dependência química com outras comorbidades psiquiátricas, abordar a dependência sem intervir também nas comorbidades denota tratamento incompleto, acarretando maior índice de recaídas, menor adesão terapêutica, maior necessidade de internações prolongadas e maior risco de suicídio ${ }^{17-24}$.

Segundo o Epidemiologic Catchment Area Study²5, cerca de metade dos dependentes de álcool e outras substâncias possuía um diagnóstico psiquiátrico adicional, sendo $26 \%$ de transtornos de humor, $28 \%$ de ansiedade e $18 \%$ de personalidade antissocial. Os resultados do National Epidemiologic Survey on Alcohol and Related Conditions ${ }^{26,27}$ demonstram associação significativa entre transtornos de humor, ansiedade e personalidade com a dependência de álcool. Além disso, outros estudos observaram maior risco de suicídio nos grupos com histórico de consumo múltiplo de substâncias, sugerindo a importância da avaliação de transtornos associados à dependência ${ }^{26-30}$.

A vulnerabilidade da população de dependentes químicos demanda tratamento especializado, que deve ser adequado às particularidades de cada caso, a depender da gravidade da dependência, da aceitação do paciente, da presença de comorbidades e do perfil sociodemográfico ${ }^{31,32}$. As formas de tratamento disponíveis vão desde o tratamento ambulatorial na atenção básica, nos Centros de Reabilitação Psicossocial e nos grupos de ajuda mútua, até a internação, seja ela voluntária ou involuntária, em hospitais gerais, hospitais psiquiátricos, clínicas especializadas e em comunidades terapêuticas ${ }^{31,33}$.

As comunidades terapêuticas consistem em um ambiente livre de SPAs, em que os pacientes são os maiores responsáveis por sua reabilitação, com um sistema estruturado por meio de normas, horários e atividades, atribuindo funções, direitos e responsabilidades aos indivíduos em tratamento ${ }^{34}$. No entanto, dado o potencial risco de agravos à saúde associado aos pacientes com dependência química, esse modelo ainda carece de adequações, como a presença de profissionais da saúde em tempo integral, planejamento para reinserção no mercado de trabalho e melhores estratégias de psicoeducação ${ }^{35}$.

Dada a alta associação de comorbidades psiquiátricas com dependência química e visando detalhar o perfil dos pacientes com dependência de SPAs, este estudo tem como objetivo analisar os dados sociodemográficos, antecedentes pessoais/familiares e a prevalência de comorbidades psiquiátricas em uma amostra de dependentes de álcool e/ou SPAs ilícitas em comunidades terapêuticas.

\section{MÉTODOS}

Trata-se de um estudo de prevalência realizado em duas comunidades terapêuticas do município de Jaci-SP, que se destinam ao tratamento de dependentes químicos do sexo masculino, maiores de 18 anos. A participação foi voluntária, sem remuneração e também sem ônus aos que não participassem. Este estudo foi aprovado pelo Comitê de Ética em Pesquisa em Seres Humanos da Faculdade de Medicina de São José do Rio Preto (CEP/Famerp) e todos os participantes assinaram o Termo de Consentimento Livre e Esclarecido.

Critérios de inclusão: preencher critérios do Manual Diagnóstico e Estatístico de Transtornos Mentais, $4^{a}$ edição (DSM-IV), explorados por meio do Mini International Neuropsychiatric Interview versão Plus (MINI Plus), assim como da Classificação Internacional das Doenças, 10a edição (CID-10), para dependência química de uma ou mais das seguintes SPAs: álcool, maconha, cocaína ou crack; tempo mínimo de cinco anos de dependência para pelo menos uma das substâncias citadas; idade entre 18 e 59 anos; sexo masculino; estar entre o $6^{\circ}$ e o $15^{\circ}$ dia de internação. 
Critérios de exclusão: preencher critérios da CID-10 para transtornos mentais e comportamentais devidos ao uso de substância - intoxicação aguda, uso nocivo para a saúde, síndrome de abstinência ou síndrome amnésica; discordância do Termo de Consentimento Livre e Esclarecido; não conseguir completar a entrevista.

Foram realizadas entrevistas clínicas por quatro médicos treinados, com aplicação de dois instrumentos: uma ficha de dados sociodemográficos/antecedentes pessoais e familiares (Anexo 1), elaborada pelos autores deste estudo, e o MINI Plus, que consiste em um questionário validado para uso no Brasil, destinado à avaliação aprofundada dos transtornos mentais ao longo da vida, na clínica e na pesquisa em psiquiatria ${ }^{36}$. O MINI Plus explora sistematicamente todos os critérios de inclusão e de exclusão e a cronologia de 23 categorias diagnósticas do DSM-IV ${ }^{36}$. Os entrevistados foram divididos em três grupos, cada um com 30 integrantes: grupos A (dependência apenas de álcool), B (dependência apenas de SPAs ilícitas) e C (dependência de álcool e SPAs ilícitas).

Os participantes foram entrevistados entre o $6^{\circ}$ e o $15^{\circ}$ dia de internação, excluindo o período imediato pós-internação, no qual a intoxicação aguda e a abstinência são mais comuns, e eliminariam o paciente da entrevista por ser critério de exclusão. Como muitos pacientes acabam sendo medicados por seus médicos assistentes logo nos primeiros dias de internação, não foram realizadas entrevistas após a segunda semana, visando evitar a ação medicamentosa plena, que geralmente se estabelece entre a terceira e a quarta semana de uso contínuo, a qual poderia reduzir os sintomas de possíveis transtornos mentais, subestimando, assim, os resultados do MINI Plus ${ }^{37}$.

Foi realizada uma análise descritiva dos dados. A comparação das frequências em cada variável, entre os grupos, foi realizada a partir da aplicação do teste de qui-quadrado clássico. O nível de significância adotado foi de 5\%. O programa utilizado para a análise foi o Prisma 6.0.

\section{RESULTADOS}

\section{Dados sociodemográficos (Tabela 1)}

A tabela 1 apresenta os dados sociodemográficos dos três grupos da pesquisa.

Houve tendência dos pacientes do grupo A de serem internados com idades mais avançadas, enquanto no grupo B observou-se o inverso. No grupo C, houve homogeneidade na distribuição das idades. Nos três grupos, o estado civil mais frequente foi o "solteiro", no entanto a prevalência de solteiros foi significativamente maior no grupo $B$ do que no grupo A.

O nível de escolaridade mais frequente foi o fundamental incompleto. Somente 10,1\% dos participantes cursaram o ensino superior e apenas 3,4\% completaram-no. A maioria dos entrevistados tinha emprego no momento da internação, exceto no grupo B. Verificou-se em todos os grupos baixa remuneração, com $70 \%$ ou mais com remuneração menor ou igual a dois salários mínimos; 37,8\% ganhavam um salário ou menos; somente 10\% tinham renda superior a três salários. Houve prevalência significativamente maior dos próprios entrevistados como maiores responsáveis pelo sustento familiar no grupo A em relação ao B.

\section{Antecedentes pessoais e familiares (Tabela 2)}

A tabela 2 apresenta os dados referentes aos antecedentes pessoais e familiares dos três grupos da pesquisa.

Mais que 70\% dos entrevistados já haviam sido internados por dependência química pelo menos três vezes. Dentre todos os pacientes, 70\% tinham história familiar de dependência química. Em relação às comorbidades clínicas, 21,1\% de todos os entrevistados apresentavam uma ou mais, e a mais citada foi a hipertensão arterial sistêmica.

\section{Aspectos relativos à dependência química (Tabela 3)}

A tabela 3 apresenta a prevalência de dependência química para cada substância investigada.

Entre todos os entrevistados, 44 (48,8\%) apresentavam mais de uma dependência química, excluindo o tabagismo. A combinação mais frequentemente encontrada foi a de maconha/cocaína, seguida por álcool/crack e álcool/maconha/cocaína.

\section{Frequência e tipos de comorbidades psiquiátricas (Figura 1)}

A figura 1 apresenta a frequência e os tipos de comorbidades psiquiátricas encontradas nos três grupos da pesquisa.

Quanto aos transtornos de humor, para o episódio depressivo maior (EDM), observaram-se prevalências totais (episódios passados e atuais) de 36,7\% no grupo A, 13,4\% no B e 30\% no C. A prevalência de transtorno depressivo maior (TDM) atual ou passado, com ou sem aspectos psicóticos (AP), nos 90 entrevistados, foi de 23,3\%. Os dados referentes aos episódios depressivos maiores induzidos por substâncias evidenciam prevalências de $16,7 \%, 23,3 \%$ e 16,7\% nos grupos $A, B$ e $C$, respectivamente. Apenas no grupo $C$ houve o diagnóstico de transtorno afetivo bipolar (TAB) tipo I e somente nos grupos B e C houve casos de episódio hipo(maníaco) induzido por substância.

Em relação aos transtornos psicóticos, apenas dois entrevistados do grupo C tiveram diagnóstico de esquizofrenia, ambos em episódio atual. Para o transtorno psicótico induzido por substância, houve prevalência de 30\% no grupo A, $60 \%$ no B e $46,7 \%$ no C, com prevalência total de $45,5 \%$. 
Tabela 1. Dados sociodemográficos

\begin{tabular}{|c|c|c|c|c|c|c|c|c|c|c|c|c|}
\hline \multirow{2}{*}{$\begin{array}{l}\text { Dados } \\
\text { sociodemográficos }\end{array}$} & \multirow{2}{*}{ Especificadores } & \multicolumn{2}{|c|}{$\begin{array}{c}\text { Grupo A } \\
\text { (30 entrevistados) }\end{array}$} & \multicolumn{2}{|c|}{$\begin{array}{c}\text { Grupo B } \\
\text { (30 entrevistados) }\end{array}$} & \multicolumn{2}{|c|}{$\begin{array}{c}\text { Grupo C } \\
\text { (30 entrevistados) }\end{array}$} & \multicolumn{2}{|c|}{$\begin{array}{c}\text { Total } \\
\text { (90 entrevistados) }\end{array}$} & \multicolumn{3}{|c|}{ Valor "p" } \\
\hline & & $\mathbf{N}^{\circ}$ & $\%$ & $\mathbf{N}^{\circ}$ & $\%$ & $\mathbf{N}^{\circ}$ & $\%$ & $\mathbf{N}^{\circ}$ & $\%$ & $A \times B$ & $A \times C$ & $B \times C$ \\
\hline \multirow{4}{*}{ Idade } & $\geq 18$ anos e $\leq 30$ anos & 1 & 3,3 & 20 & 66,7 & 6 & 20 & 27 & 30 & \multirow{4}{*}{$<0,0001$} & \multirow{4}{*}{0,0651} & \multirow{4}{*}{$<0,000$} \\
\hline & $>30$ anos e $\leq 40$ anos & 9 & 30 & 10 & 33,3 & 10 & 33,3 & 29 & 32,2 & & & \\
\hline & $>40$ anos e $\leq 50$ anos & 12 & 40 & 0 & 0 & 12 & 40 & 24 & 26,7 & & & \\
\hline & $>50$ anos e $<60$ anos & 8 & 26,7 & 0 & 0 & 2 & 6,7 & 10 & 11,1 & & & \\
\hline \multirow{6}{*}{ Estado civil } & Solteiro & 11 & 36,7 & 24 & 80 & 16 & 53,3 & 51 & 56,7 & \multirow{6}{*}{0,0224} & \multirow{6}{*}{0,0526} & \multirow{6}{*}{0,0431} \\
\hline & Casado & 8 & 26,7 & 3 & 10 & 3 & 10 & 14 & 15,5 & & & \\
\hline & Divorciado & 6 & 20 & 3 & 10 & 1 & 3,3 & 10 & 11,1 & & & \\
\hline & Separado & 3 & 10 & 0 & 0 & 5 & 16,7 & 08 & 8,9 & & & \\
\hline & Viúvo & 1 & 3,3 & 0 & 0 & 0 & 0 & 1 & 1,1 & & & \\
\hline & União consensual & 1 & 3,3 & 0 & 0 & 5 & 16,7 & 6 & 6,7 & & & \\
\hline \multirow{3}{*}{ Prole } & Sim & 21 & 70 & 15 & 50 & 19 & 63,3 & 55 & 61,1 & \multirow{3}{*}{0,2444} & \multirow{3}{*}{0,8607} & \multirow{3}{*}{0,4906} \\
\hline & Não & 9 & 30 & 15 & 50 & 11 & 36,7 & 35 & 38,9 & & & \\
\hline & $\begin{array}{l}\text { Média de filhos/ } \\
\text { entrevistado }\end{array}$ & 2,23 & $\emptyset$ & 1,46 & $\emptyset$ & 2,0 & $\emptyset$ & 1,89 & $\emptyset$ & & & \\
\hline \multirow{9}{*}{ Escolaridade } & Nunca estudou & 0 & 0 & 0 & 0 & 0 & 0 & 0 & 0 & \multirow{9}{*}{0,3106} & & \\
\hline & Fundamental incompleto & 18 & 60 & 12 & 40 & 16 & 53,3 & 46 & 51,1 & & & \\
\hline & Fundamental completo & 4 & 13,4 & 4 & 13,3 & 5 & 16,6 & 13 & 14,4 & & & \\
\hline & Médio incompleto & 1 & 3,3 & 5 & 16,7 & 3 & 10 & 9 & 10 & & & \\
\hline & Médio completo & 5 & 16,7 & 6 & 20 & 2 & 6,7 & 13 & 14,4 & & 0,6721 & 0,3710 \\
\hline & Superior incompleto & 1 & 3,3 & 3 & 10 & 2 & 6,7 & 6 & 6,7 & & & \\
\hline & Superior completo & 1 & 3,3 & 0 & 0 & 2 & 6,7 & 3 & 3,4 & & & \\
\hline & Curso técnico incompleto & 0 & 0 & 0 & 0 & 0 & 0 & 0 & 0 & & & \\
\hline & Curso técnico completo & 0 & 0 & 0 & 0 & 0 & 0 & 0 & 0 & & & \\
\hline & Empregado & 17 & 56,7 & 12 & 40 & 21 & 70 & 50 & 55,5 & & & \\
\hline Profissão & Desempregado & 12 & 40 & 16 & 53,3 & 6 & 20 & 34 & 37,8 & 0,4134 & 0,1808 & 0,3899 \\
\hline & Outros & 1 & 3,3 & 2 & 6,7 & 3 & 10 & 6 & 6,7 & & & \\
\hline & $\leq 1$ salário mínimo & 10 & 33,3 & 12 & 40 & 12 & 40 & 34 & 37,8 & & & \\
\hline Renda pessoal (do & $>1$ e $\leq 2$ salários mínimos & 11 & 36,7 & 12 & 40 & 9 & 30 & 32 & 35,5 & 07007 & 08004 & 08180 \\
\hline último que teve) & $>2$ e $\leq 3$ salários mínimos & 7 & 23,3 & 4 & 13,3 & 4 & 13,3 & 15 & 16,7 & 0,1901 & 0,0004 & 0,0100 \\
\hline & > 3 salários mínimos & 2 & 6,7 & 2 & 6,7 & 5 & 16,7 & 9 & 10 & & & \\
\hline & 0 próprio entrevistado & 18 & 60 & 5 & 16,7 & 18 & 60 & 41 & 45,5 & & & \\
\hline & Pai do entrevistado & 1 & 3,3 & 7 & 23,3 & 2 & 6,7 & 10 & 11,2 & & & \\
\hline & Mãe do entrevistado & 5 & 16,6 & 10 & 33,3 & 4 & 13,3 & 19 & 21,2 & & & \\
\hline Maior responsável pelo & Cônjuge do entrevistado & 2 & 6,7 & 1 & 3,3 & 4 & 13,3 & 7 & 7,8 & $000 \Delta 0$ & 7312 & 00165 \\
\hline sustento da família & Irmão(ã) do entrevistado & 2 & 6,7 & 2 & 6,7 & 0 & 0 & 4 & 4,4 & 0,0040 & 0,1340 & (0, \\
\hline & Filho(a) do entrevistado & 2 & 6,7 & 0 & 0 & 1 & 3,3 & 3 & 3,3 & & & \\
\hline & Avô(ó) do entrevistado & 0 & 0 & 2 & 6,7 & 0 & 0 & 2 & 2,2 & & & \\
\hline & Outros & 0 & 0 & 3 & 10 & 1 & 3,4 & 4 & 4,4 & & & \\
\hline & Católico & 21 & 70 & 15 & 50 & 15 & 50 & 51 & 56,7 & & & \\
\hline & Evangélico & 7 & 23,3 & 7 & 23,3 & 8 & 26,7 & 22 & 24,4 & & & \\
\hline Religião & Espírita & 1 & 3,3 & 2 & 6,7 & 0 & 0 & 3 & 3,4 & 0,1721 & 0,1563 & 0,5467 \\
\hline & Ateu & 0 & 0 & 0 & 0 & 1 & 3,3 & 1 & 1,1 & & & \\
\hline & Outras & 1 & 3,3 & 6 & 20 & 6 & 20 & 13 & 14,4 & & & \\
\hline
\end{tabular}


Tabela 2. Antecedentes pessoais e familiares

\begin{tabular}{|c|c|c|c|c|c|c|c|c|c|c|c|c|}
\hline \multirow{2}{*}{$\begin{array}{l}\text { Antecedentes pessoais } \mathrm{e} \\
\text { familiares }\end{array}$} & \multirow{2}{*}{ Especificadores } & \multicolumn{2}{|c|}{$\begin{array}{c}\text { Grupo A } \\
\text { (30 entrevistados) }\end{array}$} & \multicolumn{2}{|c|}{$\begin{array}{c}\text { Grupo B } \\
\text { (30 entrevistados) }\end{array}$} & \multicolumn{2}{|c|}{$\begin{array}{c}\text { Grupo } \\
\text { (30 entrevistados) }\end{array}$} & \multicolumn{2}{|c|}{$\begin{array}{c}\text { Total } \\
\text { (90 entrevistados) }\end{array}$} & \multicolumn{3}{|c|}{ Valor "p" } \\
\hline & & $\mathbf{N}^{\circ}$ & $\%$ & $\mathrm{~N}^{\circ}$ & $\%$ & $\mathbf{N}^{\circ}$ & $\%$ & $\mathrm{~N}^{\circ}$ & $\%$ & $A \times B$ & $\operatorname{AxC}$ & $B \times C$ \\
\hline \multirow{6}{*}{$\begin{array}{l}\text { Número de internaçöes } \\
\text { por dependência química }\end{array}$} & 1 vez (1a internação) & 10 & 33,3 & 15 & 50 & 12 & 40 & 37 & 41,1 & \multirow{6}{*}{0,6083} & \multirow{6}{*}{0,7598} & \multirow{6}{*}{0,4552} \\
\hline & 2 vezes & 9 & 30 & 6 & 20 & 9 & 30 & 24 & 26,6 & & & \\
\hline & 3 vezes & 3 & 10 & 3 & 10 & 2 & 6,7 & 8 & 8,8 & & & \\
\hline & $4-5$ vezes & 4 & 13,3 & 4 & 13,3 & 3 & 10 & 11 & 12,2 & & & \\
\hline & $6-10$ vezes & 2 & 6,7 & 2 & 6,7 & 1 & 3,3 & 5 & 5,5 & & & \\
\hline & $>10$ vezes & 2 & 6,7 & 0 & 0 & 3 & 10 & 5 & 5,5 & & & \\
\hline \multirow{9}{*}{$\begin{array}{l}\text { História familiar de } \\
\text { dependência química }\end{array}$} & Pai & 13 & & 8 & & 8 & & 29 & & \multirow{9}{*}{0,0951} & \multirow{9}{*}{0,5102} & \multirow{9}{*}{0,4191} \\
\hline & Mãe & 0 & & 3 & & 0 & & 3 & & & & \\
\hline & Irmão(ã)/(ãos) & 10 & & 8 & & 10 & & 28 & & & & \\
\hline & Avô(ó)/(ós) & 0 & $\emptyset$ & 1 & $\emptyset$ & 2 & $\emptyset$ & 3 & $\emptyset$ & & & \\
\hline & Filho(a)/(os)/(as) & 0 & & 0 & & 0 & & 0 & & & & \\
\hline & $\mathrm{Tio}(\mathrm{a}) /(0 \mathrm{~s}) /(\mathrm{as})$ & 4 & & 4 & & 4 & & 12 & & & & \\
\hline & Outros & 1 & & 7 & & 3 & & 11 & & & & \\
\hline & Nega & 11 & 36,6 & 7 & 23,3 & 9 & 30 & 27 & 30 & & & \\
\hline & Confirma 1 ou mais & 19 & 63,4 & 23 & 76,7 & 21 & 70 & 63 & 70 & & & \\
\hline \multirow{13}{*}{$\begin{array}{l}\text { Comorbidades clínicas } \\
\text { principais }\end{array}$} & Hipertensão arterial sistêmica & 6 & & 2 & & 2 & & 10 & & \multirow{13}{*}{0,1045} & \multirow{13}{*}{0,1001} & \multirow{13}{*}{0,1671} \\
\hline & Diabetes mellitus & 1 & & 0 & & 3 & & 4 & & & & \\
\hline & Dislipidemia & 0 & & 1 & & 0 & & 1 & & & & \\
\hline & Cirrose hepática & 0 & & 0 & & 0 & & 0 & & & & \\
\hline & $\begin{array}{l}\text { Doença pulmonar obstrutiva } \\
\text { crônica }\end{array}$ & 0 & & 0 & & 0 & & 0 & & & & \\
\hline & Asma & 0 & $\emptyset$ & 0 & $\emptyset$ & 0 & $\emptyset$ & 0 & $\emptyset$ & & & \\
\hline & Anemia & 1 & & 0 & & 0 & & 0 & & & & \\
\hline & HIV & 0 & & 0 & & 0 & & 0 & & & & \\
\hline & Hepatites B ou C & 0 & & 0 & & 0 & & 0 & & & & \\
\hline & $\begin{array}{l}\text { Outras doenças sexualmente } \\
\text { transmissiveis }\end{array}$ & 0 & & 0 & & 0 & & 0 & & & & \\
\hline & Outras & 7 & & 2 & & 1 & & 10 & & & & \\
\hline & Nenhuma & 19 & 63,4 & 27 & 90 & 25 & 83,4 & 71 & 78,9 & & & \\
\hline & Confirma 1 ou mais & 11 & 36,6 & 03 & 10 & 05 & 16,6 & 19 & 21,1 & & & \\
\hline
\end{tabular}

$\emptyset$ Ø: não se aplica cálculo de percentual.

Tabela 3. Prevalência de dependência química

\begin{tabular}{lcc}
\hline Substâncias psicoativas - dependência química (atual ou prévia) & Número de dependentes químicos entre os 90 entrevistados & Percentual de dependentes químicos entre os 90 entrevistados \\
\hline Tabaco & 74 & $82,2 \%$ \\
Álcool & 60 & $67,7 \%$ \\
Cocaína & 43 & $47,7 \%$ \\
Crack & 39 & $43,3 \%$ \\
Maconha & 38 & $42,2 \%$ \\
Inalantes & 6 & $6,6 \%$ \\
Alucinógenos & 3 & $3,3 \%$ \\
Anfetaminas & 2 & $2,2 \%$ \\
Benzodiazepínicos & 1 & $1,1 \%$ \\
Opioides & 0 & Zero \\
\hline
\end{tabular}




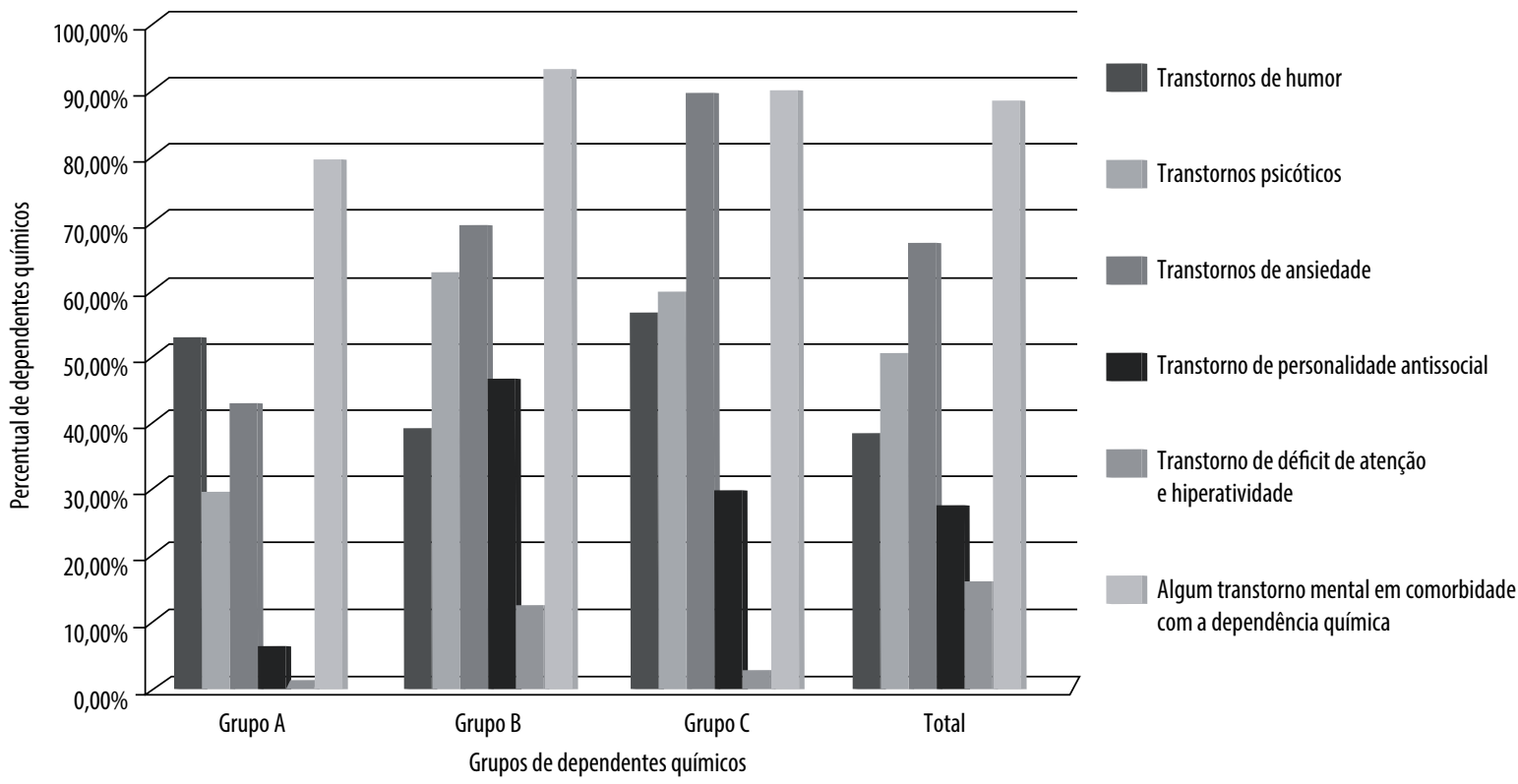

Figura 1. Gráfico de comorbidades psiquiátricas em dependentes químicos.

Para os transtornos de ansiedade, dois entrevistados no grupo A (6,7\%) e um no B (3,3\%) preencheram critérios para transtorno de ansiedade generalizada (TAG) atual. Para o TAG induzido por substância atual, houve maior prevalência, com cinco no grupo A e B (16,7\%) e um no C (3,3\%). Tal prevalência foi significativamente maior no grupo $A$ do que no $C(p=0,0452)$ e no $B$ em relação ao $C(p=0,0452)$. Seis entrevistados no grupo B (20\%) foram diagnosticados com transtorno de ansiedade com ataques de pânico induzidos por substância, enquanto apenas um no grupo C (3,3\%). Dentre os 90 entrevistados, oito (8,9\%) foram detectados com fobia social e $10(11,1 \%)$ com fobia específica. Houve quatro casos de transtorno de estresse pós-traumático (TEPT), todos no grupo C (13,3\%).

Diagnosticou-se o transtorno da personalidade antissocial na vida inteira em dois entrevistados no grupo $A(6,7 \%)$, 14 no B $(46,7 \%)$ e nove no $C(30 \%)$, totalizando 25 entre todos os entrevistados (27,8\%). Esse transtorno foi significativamente mais frequente no grupo $B$ do que no $A(p=0,0005)$ e mais no $C$ do que no $A(p=0,0195)$. Foi ainda mais frequente no $B$ do que no $C$, no entanto essa última associação não teve validade estatística $(p=0,1843)$.

O transtorno de déficit de atenção e hiperatividade (TDAH) adulto atual foi detectado em quatro pacientes do grupo B (13,3\%) e em um do C (3,3\%). A frequência de TDAH foi significativamente superior no grupo $B$ em relação ao $A$ $(p=0,0384)$.

Somente seis entrevistados no grupo A (20\%), dois no $B(6,7 \%)$ e três no $C(10 \%)$ não tiveram nenhum transtorno mental detectado pelo MINI Plus, excluindo a dependência química. Portanto, em $88,8 \%$ da amostra encontrou-se algum transtorno mental em comorbidade com a dependência química.

\section{Perfis dos entrevistados por grupos (Tabela 4)}

A tabela 4 apresenta uma síntese por grupos dos perfis dos entrevistados, levando em consideração os dados sociodemográficos, os antecedentes pessoais e familiares e as comorbidades psiquiátricas.

Tabela 4. Perfis dos entrevistados por grupos

\begin{tabular}{ll}
\hline Grupos & Perfil \\
\hline Grupo A & Idade avançada na internação \\
& Maior número de filhos \\
& Com emprego \\
& Maiores responsáveis pelo sustento da família \\
& Diagnosticados com transtorno depressivo maior \\
\hline Grupo B & Idade precoce na internação \\
& Solteiros \\
& Desempregados \\
& Pais como principais provedores da família \\
& Diagnosticados com transtorno psicótico induzido por substância, \\
& transtornos de ansiedade, transtorno de personalidade antissocial e TDAH \\
\hline GrupoC & Idade avançada na internação \\
& Maior número de filhos \\
& Com emprego \\
& Maiores responsáveis pelo sustento da família \\
& Diagnosticados com transtorno psicótico induzido por substância, \\
& transtornos de ansiedade e transtorno de personalidade antissocial \\
\hline
\end{tabular}

\section{DISCUSSÃO}

\section{Dados sociodemográficos}

No presente estudo, observou-se que o uso de SPAs ilícitas levou à internação mais precocemente do que o uso isolado de álcool. Borini et al. ${ }^{38}$ verificaram achado similar em 382 pacientes masculinos internados por uso de SPAs ilícitas, no qual cerca de metade tinha idade abaixo de 20 anos. Sabe-se que entre as SPAs ilícitas está o crack, cujos primeiros sinto- 
mas surgem de 10 a 15 segundos após sua utilização, sendo ainda de rápida eliminação, o que acarreta o uso compulsivo, podendo demandar internação precoce ${ }^{39}$.

O envolvimento com SPAs ilícitas e álcool pode dificultar a constituição de família. Ao analisarem 350 prontuários de dependentes químicos, Capistrano et al. ${ }^{6}$ verificaram alto percentual de solteiros $(39,4 \%)$, como o encontrado neste estudo (56,7\%). Silva et al. ${ }^{1}$ encontraram prevalência de separação conjugal de 80\% e consideraram a adição um fator de risco para a desestruturação familiar. É plausível que o grupo $B$ apresente maior prevalência de solteiros em relação ao A, por ser o grupo constituído por mais adultos jovens. A dependência isolada de álcool possibilita manter um padrão de vida mais organizado até idades mais avançadas, permitindo a constituição de família ${ }^{40,41}$. Já nos usuários de SPAs ilícitas apenas, as dificuldades sociais pronunciam-se mais precocemente, dificultando a constituição familiar ${ }^{40,41}$.

No presente estudo, a maioria dos entrevistados só cursou até o ensino fundamental. Dados da literatura sugerem que a baixa escolaridade não é apenas consequência do uso de SPAs, mas também causa, pois a menor escolaridade resulta em maior dificuldade para a inserção no mercado de trabalho, acarretando marginalização e envolvimento mais frequente com SPAs ${ }^{42,43}$. Bahls e Ingbermann ${ }^{44}$ encontraram um início precoce de dificuldades e abandono escolar podendo prenunciar o abuso de drogas.

Os resultados demonstram que mais da metade dos os entrevistados estava empregada no momento da internação. Todavia, muitos desses empregos não eram formais, e sim trabalhos intermitentes, pois o estreitamento de repertório imposto pelas SPAs dificulta a regularidade no trabaIho ${ }^{45}$. Segundo o National Household Survey on Drug Abuse ${ }^{46}$, dos 11,8 milhões de adultos usuários de drogas no ano 2000, 9,1 milhões (77\%) tinham algum emprego.

Verificou-se que 37,8\% dos participantes tinham renda menor ou igual a um salário mínimo. Sadock e Sadock ${ }^{47}$ sustentam que a menor escolaridade e os níveis mais baixos de renda são fatores de risco para a dependência química.

A alta prevalência de catolicismo no Brasil, conforme elucidado no "Novo mapa das religiões"48, explica o fato de a maior parte dos entrevistados serem católicos. Além disso, o fato de o presente estudo ter sido realizado em duas comunidades católicas pode ter tornando mais confortável para alguns entrevistados assumir o catolicismo ${ }^{49}$.

\section{Antecedentes pessoais e familiares}

Os resultados corroboram a alta prevalência de recaída evidenciada na literatura ${ }^{50}$, já que para todos os grupos estudados pelo menos 50\% dos entrevistados já tinham mais de uma internação por dependência química, denotando recaídas. Analogamente, Zenelatto ${ }^{51}$ encontrou alta incidência de recaída nos primeiros 90 dias e até mesmo após anos de abstinência.
Os dados apontam alta prevalência de história familiar de dependência química (70\%), sendo o pai o familiar mais citado. Conforme discutido por Canavez et al. ${ }^{52}$, a predisposição genética e o convívio com pais e irmãos usuários de SPAs são fatores de risco para a dependência química. Figlie et al. ${ }^{53}$ encontraram prevalência paterna de 67\% para o uso de SPAs.

\section{Aspectos relativos à dependência química}

O fato de o tabaco e o álcool serem substâncias lícitas, de baixo custo e bem aceitas socialmente pode justificar suas maiores prevalências ${ }^{54}$. Além disso, o álcool e o tabaco são mais prejudiciais em longo prazo, enquanto a cocaína e o crack trazem debilidade física e psíquica, envolvimento com violência e internação mais precoces ${ }^{55}$. Ribeiro et al. ${ }^{55}$ verificaram que, após cinco anos de seguimento de 124 usuários de cocaína/crack, 17,6\% morreram, sendo homicídio a causa mais prevalente, seguida de infecção pelo vírus da imunodeficiência adquirida.

As prevalências de dependência de maconha, cocaína e crack foram próximas, o que pode ser explicado pela progressão comum do uso dessas substâncias: início com maconha, seguida pela cocaína e o $\operatorname{crack}^{56}$. Similarmente, Sanchez e Nappo $^{57}$ constataram 31 usuários ou ex-usuários de crack seguindo essa mesma escalada típica da dependência química.

\section{Comorbidades psiquiátricas}

A prevalência de EDM e TDM foi semelhante nos grupos A e C e superior ao B. Pode-se inferir que o álcool é um importante fator para a similaridade entre A e C quanto ao TDM, conforme revisão de 2011, que avaliou a associação entre o uso de álcool e a depressão, evidenciando que, quando um deles está presente, se dobra o risco para o outro ${ }^{58}$. Já para o EDM induzido por substância, as prevalências de $\mathrm{A}$ e $\mathrm{C}$ foram iguais e inferiores à do B. A prevalência de EDM foi superior à de TDM, pois nem todos os episódios depressivos eram unipolares.

Foi diagnosticado TAB apenas nos grupos B e C, reforçando uma possível associação entre tal diagnóstico e o uso de SPAs ilícitas. Como prováveis causas, Ribeiro et al. ${ }^{59}$ apontam a cocaína como potencializadora dos estados maníacos e o consumo de maconha aumentando o risco, a intensidade e o tempo de duração de uma crise. Em contrapartida, Regier et al. ${ }^{25}$ encontraram prevalência de dependência de SPAs ilícitas associada ao TAB de $27 \%$, enquanto nos etilistas foi de $31 \%$.

A prevalência de esquizofrenia foi de $2,2 \%$, mais que o triplo da população geral $\left(0,3 \%\right.$ a 0,7\%) ${ }^{60}$. O transtorno psicótico induzido por substância acometeu mais de 40\% dos entrevistados, principalmente no grupo C (> 50\%). O grupo A teve a menor prevalência, sugerindo que as drogas ilícitas possam induzir sintomas psicóticos mais facilmente do que o álcool sozinho. As diretrizes da Associação Brasileira de Estudos do Álcool e outras Drogas ${ }^{17}$ demonstram que a esqui- 
zofrenia associada ao abuso de substâncias tem prognóstico pior do que um desses transtornos isoladamente.

Quanto aos transtornos de ansiedade, observou-se certa homogeneidade entre os grupos B e C e menor prevalência no A. O perfil de maior delinquência encontrado nos usuários de drogas ilícitas os expõe a situações de pressão e perigo, favorecendo o advento de transtornos de ansiedade ${ }^{7,17,61}$.

A prevalência de transtorno da personalidade antissocial na vida inteira $(26,7 \%)$, sobretudo considerando as prevalências dos grupos B $(46,7 \%)$ e C (30\%), foi muito superior à da população geral $(0,2 \%-3,3 \%)^{60}$. Infere-se uma associação desse transtorno com o uso de SPAs, sobretudo ilícitas, visto que os grupos B e C tiveram prevalências similares e altas, enquanto o grupo A teve prevalência menor (6,7\%). A personalidade antissocial favorece comportamentos desviantes, com maior envolvimento em criminalidade e uso de SPAs ${ }^{27}$. Chalub e Telles ${ }^{7}$ encontraram alta proporção de atos violentos quando álcool ou drogas ilícitas estavam presentes entre agressores ou vítimas.

Os resultados sugerem uma associação entre o uso de SPAs ilícitas e o TDAH: quatro pacientes do grupo B e um do $C$ apresentaram diagnóstico de TDAH, enquanto nenhum do grupo A apresentou. Um estudo com 120 indivíduos com TDAH na infância versus 268 sem a doença evidenciou que a prevalência de uso de SPAs na vida adulta foi superior nos pacientes com TDAH (52\% versus $27 \%)^{62}$. Entretanto, essa relação não existia quando se considerava o uso isolado de álcool, mas estava presente no uso isolado de drogas ilícitas ou de SPAs ilícitas e álcool ${ }^{62}$.

A elevada prevalência de comorbidades psiquiátricas encontrada nos participantes deste estudo $(88,8 \%)$, superior à da população geral $(56 \%)^{63}$, pode decorrer de todos os entrevistados estarem internados e preencherem critérios para dependência química, sendo, portanto, mais graves. Capistrano et al. ${ }^{6}$ verificaram prevalência de comorbidades psiquiátricas em dependentes químicos de 13\%, significativamente inferior à encontrada neste estudo. Já outros trabalhos demonstraram taxas mais altas de comorbidades psiquiátricas, de aproximadamente 50\% e 83,9\% 25,29 .

É importante destacar ser o MINI Plus um instrumento desenhado para o screening de transtornos psiquiátricos na atenção primária ${ }^{36}$. Portanto, alguns resultados podem estar superestimados, uma vez que ele foi utilizado em um cenário de comunidades terapêuticas. Ademais, foram pesquisados diagnósticos de difícil realização, como o de TDAH no adulto e o de transtorno da personalidade antissocial, podendo haver falsos-positivos, o que pode levar à maior sobreposição de diagnósticos. Assim sendo, as discussões elaboradas merecem ponderação.

\section{Perfis dos entrevistados por grupos}

Houve semelhança em relação aos dados sociodemográficos/antecedentes pessoais e familiares entre os grupos A e C.
Pressupõe-se que isso se deva ao fato de os integrantes do grupo C, na maioria das vezes, iniciarem a dependência pelo álcool e posteriormente evoluírem para dependência de SPAs ilícitas. Esse tempo seria suficiente para constituir família e estabelecer vínculo empregatício.

Por outro lado, quanto às comorbidades psiquiátricas, observa-se maior similaridade entre os grupos B e C: altas prevalências de transtorno de personalidade antissocial, transtorno psicótico induzido por substância, transtornos de ansiedade, além da presença de TDAH. Tal fato sugere que as SPAs ilícitas sejam as determinantes para o perfil de comorbidades psiquiátricas, com exceção do TDM, para o qual a dependência de álcool parece ser mais significativa.

\section{CONCLUSÃO}

A população estudada apresenta vulnerabilidades como baixa escolaridade, remuneração insuficiente, instabilidade conjugal, história familiar de dependência química e alta prevalência de comorbidades psiquiátricas. Foi possível traçar perfis particulares para cada grupo de dependentes químicos, observando-se diferenças significativas entre eles. Tal panorama evidencia a necessidade de atenção individualizada para o tratamento da dependência química e suas comorbidades, além de ações de prevenção.

Dada à fragilidade sociodemográfica e a alta prevalência de comorbidades verificada, sugere-se que os membros das equipes das comunidades terapêuticas poderiam aplicar escalas de risco de suicídio, depressão e ansiedade durante o período de tratamento, assim como buscar vulnerabilidades sociodemográficas peculiares a cada caso, adequando o tratamento às necesidades de cada paciente. É também fundamental direcionar esses indivíduos para tratamentos especializados pós-alta, para evitar recaídas e novas hospitalizações.

Faz-se necessária a replicação deste estudo em amostras maiores e em amostra do sexo feminino, assim como a comparação com dependentes químicos em tratamento ambulatorial e com a população geral. Por fim, seria importante realizar estudos prospectivos que levassem em consideração fatores sociodemográficos, com o intuito de compreender a dinâmica do surgimento das comorbidades psiquiátricas na dependência química.

\section{CONTRIBUIÇÕES INDIVIDUAIS}

Rafael Vinícius Danieli, Mírian Brusadelli Macedo Ferreira, Leonardo Nunes de Castro Oliveira e Julia Melloni Nogueira - Responsáveis pela concepção e desenho do estudo; coleta, análise e interpretação dos dados; elaboração 
do artigo e revisão crítica do conteúdo intelectual; versão final para publicação aprovada.

Emirene Maria Trevisan Navarro da Cruz e Gerardo Maria de Araújo Filho - Responsáveis pelo desenho do estudo; análise e interpretação dos dados; revisão crítica do conteúdo intelectual; versão final para publicação aprovada.

\section{CONFLITOS DE INTERESSE}

Todos os autores deste estudo declaram inexistência de quaisquer conflitos de interesse.

\section{AGRADECIMENTOS}

Agradecemos à Associação São Francisco de Assis na Providência de Deus, por ter disponibilizado a amostra utilizada.

Agradecemos à Dra. Lilian Castiglione, pelo auxílio referente à análise estatística dos dados.

\section{REFERÊNCIAS}

1. Silva LHP, Borba LO, Paes MR, Guimarães AN, Mantovani MF, Maftum MA. Perfil dos dependentes químicos atendidos em uma unidade de reabilitação de um hospital psiquiátrico. Esc Anna Nery. 2010;14(3):585-90.

2. Quinderé PHD, Tófoli LF. Análise do perfil epidemiológico dos clientes do Centro de Atenção Psicossocial para Álcool e outras Drogas (CAPS-AD) de Sobral-CE. SANARE. 2005/2007;6(2):62-6

3. Redonnet B, Chollet A, Fombonne E, Bowes L, Melchior M. Tobacco, alcohol, cannabis and other illegal drug use among young adults: the socioeconomic context. Drug Alcohol Depend. 2012;121(3):231-9.

4. Centro Brasileiro de Informações sobre Drogas Psicotrópicas (CEBRID). II levantamento domiciliar sobre 0 uso de drogas psicotrópicas no Brasil: estudo envolvendo as 108 maiores cidades do país - 2005. Brasília: Secretaria Nacional Antidrogas; 2006. 468p.

5. United Nations Office on Drugs and Crime. World Drug Report 2015. New York: United Nations; 2015. 118p.

6. Capistrano FC, Ferreira ACZ, Silva TL, Kalinke LP, Maftum MA. Perfil sociodemográfico e clínico de dependentes químicos em tratamento: análise de prontuários. Esc Anna Nery. $2013 ; 17(2): 234-41$

7. Chalub M, Telles LEB. Álcool, drogas e crime. Rev Bras Psiquiatr. 2006;28(Supl II):S69-73.

8. Storr CL, Accornero VH, Crum RM. Profiles of current disruptive behavior: association with recent drug consumption among adolescents. Addict Behav. 2007;32(2):248-64.

9. Ferreira Filho OF, Turchi MD, Laranjeira R, Castelo A. Perfil sociodemográfico e de padrões de uso entre dependentes de cocaína hospitalizados. Rev Saúde Pública. 2003;37(6):751-9.

10. Guimarães CF, Santos DVV, Freitas RC, Araujo RB. Perfil do usuário de crack e fatores relacionados à criminalidade em unidade de internação para desintoxicação no Hospital Psiquiátrico São Pedro de Porto Alegre (RS). Rev Psiquiatr Rio Gd Sul. 2008;30(2):101-8.

11. Tiet QQ, Ilgen MA, Byrnes HF, Moos RH. Suicide attempts among substance use disorder patients: an initial step toward a decision tree for suicide management. Alcohol Clin Exp Res. 2006:30(6):998-1005.

12. Duailibi LB, Ribeiro M, Laranjeira R. Perfil dos usuários de cocaína e crack no Brasil. Cad Saúde Pública. 2008:24(Supl 4):S545-57.

13. Elbreder MF, Laranjeira R, Siqueira MM, Barbosa DA. Perfil das mulheres usuárias de álcoo em ambulatório especializado em dependência química. J Bras Psiquiatr. 2008:57(1):9-15.
14. Horta RG, Horta BL, Rosset AP, Horta CL. Perfil dos usuários de crack que buscam atendimento em centro de atenção psicossocial. Cad Saúde Pública. 2011;27(11):2263-70.

15. Jungerman FS, Laranjeira R. Characteristics of cannabis users seeking treatment in São Paulo, Brazil. Rev Panam Salud Publica. 2008;23(6):384-93.

16. Esper LH, Corradi-Webster CM, Carvalho AMP, Furtado EF. Mulheres em tratamento ambulatorial por abuso de álcool: características sociodemográficas e clínicas. Rev Gaúcha Enferm. 2013;34(2):93-101.

17. Zaleski M, Laranjeira RR, Roselli ACP, Ratto L, Romano M, Alves HNP, et al. Diretrizes da Associação Brasileira de Estudos do Álcool e outras Drogas (ABEAD) para o diagnóstico e tratamento de comorbidades psiquiátricas e dependência de álcool e outras substâncias. Rev Bras Psiquiatr. 2006;28(2):142-8

18. Alves H, Kessler F, Ratto LRC. Comorbidade: uso de álcool e outros transtornos psiquiátricos. Rev Bras Psiquiatr. 2004;26(Supl 1):51-3.

19. Demetrovics Z. Co-morbidity of drug addiction: an analysis of epidemiological data and possible etiological models. Addict Res Theory. 2009;17(4):420-31.

20. Jané-Llopis E, Matytsina I. Mental health and alcohol, drugs and tobacco: a review of the comorbidity between mental disorders and the use of alcohol, tobacco and illicit drugs. Drug Alcohol Rev. 2006;25(6):515-36.

21. Lai HM, Huang QR. Comorbidity of mental disorders and alcohol- and drug-use disorders: analysis of New South Wales inpatient data. Drug Alcohol Rev. 2009;28(3):235-42.

22. Compton WM, Thomas YF, Stinson FS, Grant BF. Prevalence, correlates, disability, and comorbidity of DSM-IV drug abuse and dependence in the United States: results from the national epidemiologic survey on alcohol and related conditions. Arch Gen Psychiatry. 2007;64(5):566-76

23. Schuckit MA. Comorbidity between substance use disorders and psychiatric conditions. Addiction. 2006;101 Suppl 1:76-88.

24. Swendsen J, Conway KP, Degenhardt L, Glantz M, Jin R, Merikangas KR, et al. Mental disorders as risk factors for substance use, abuse and dependence: results from the 10-year follow-up of the National Comorbidity Survey. Addiction. 2010;105(6):1117-28.

25. Regier DA, Farmer ME, Rae DS, Locke BZ, Keith SJ, Judd LL, et al. Comorbidity of mental disorders with alcohol and other drug abuse. Results from the Epidemiologic Catchment Area (ECA) Study. JAMA. 1990;264(19):2511-8.

26. Hasin DS, Stinson FS, Ogburn E, Grant BF. Prevalence, correlates, disability, and comorbidity of DSM-IV alcohol abuse and dependence in the United States: results from the National Epidemiologic Survey on Alcohol and Related Conditions. Arch Gen Psychiatry. 2007;64(7):830-42

27. Scheffer M, Pasa GG, Almeida RMM. Dependência de álcool, cocaína e crack e transtornos psiquiátricos. Psic Teor Pesq. 2010;26(3):533-41.

28. Hess ARB, Almeida RMM, Moraes AL. Comorbidades psiquiátricas em dependentes químicos em abstinência em ambiente protegido. Estud Psicol. 2012;17(1):171-8.

29. Silva CR, Kolling NM, Carvalho JCN, Cunha SM, Kristensen CH. Comorbidade psiquiátrica em dependentes de cocaína/crack e alcoolistas: um estudo exploratório. Aletheia. 2009;30:101-12

30. Leal E, Delgado PGG, Mann R, Strike C, Brands B, Khenti A. Estudo de comorbidade: sofrimento psíquico e abuso de drogas em pessoas em centros de tratamento, Macaé - Brasil. Texto Contexto Enferm. 2012;21:96-104.

31. Diehl A, Cordeiro DC, Laranjeira R. Dependência química: prevenção, tratamento e políticas públicas. Porto Alegre: Artmed; 2011

32. Najavits LM, Hien D. Helping vulnerable populations: a comprehensive review of the treatment outcome literature on substance use disorder and PTSD. J Clin Psychol. 2013;69(5):433-79.

33. Secretaria Nacional de Políticas sobre Drogas. Ministério da Justiça e Cidadania. SUPERA: Modalidades de tratamento e encaminhamento. Brasília: SENAD; 2017. 144p.

34. Sabino SDM, Cazenave SOS. Comunidades terapêuticas como forma de tratamento para a dependência de substâncias psicoativas. Estud Psicol. 2005;22(2):167-74.

35. Degenhardt L, Hall W. Extent of illicit drug use and dependence, and their contribution to the global burden of disease. Lancet. 2012;379(9810):55-70.

36. Amorim P. Mini International Neuropsychiatric Interview (MINI): validação de entrevista breve para diagnóstico de transtornos mentais. Rev Bras Psiquiatr. 2000;22(3):106-15. 
37. Spanemberg L, Caldieraro MAK, Vares EA, Fleck MPA. Depressão maior e distimia. In: Cordioli AV, editor. Psicofármacos: consulta rápida. Porto Alegre: Artmed; 2011. p. 399-412.

38. Borini P, Guimarães RC, Borini SB. Usuários de drogas ilícitas internados em hospital psiquiátrico: padrões de uso e aspectos demográficos e epidemiológicos. J Bras Psiquiatr. 2003;52(3):171-9.

39. Secretaria Nacional de Políticas sobre Drogas. Ministério da Justiça e Cidadania. SUPERA: Efeitos das substâncias psicoativas. Brasília: SENAD; 2017. 146p.

40. Silveira CM, Viana MC, Siu ER, Andrade AG, Anthony JC, Andrade LH. Sociodemographic correlates of transitions from alcohol use to disorders and remission in the São Paulo megacity mental health survey, Brazil. Alcohol Alcohol. 2011;46(3):324-32.

41. Gabbard G0. Transtornos relacionados a substâncias e transtornos da alimentação. In: Gabbard G0, editor. Psiquiatria psicodinâmica na prática clínica. Porto Alegre: Artmed; 2007. p. 259-82.

42. Roehrs H, Lenardt MH, Maftum MA. Práticas culturais familiares e 0 uso de drogas psicoativas pelos adolescentes reflexão teórica. Esc Anna Nery. 2008;12(2):353-7.

43. Pechansky F, Szobot CM, Scivoletto S. Uso de álcool entre adolescentes: conceitos, características epidemiológicas e fatores etiopatogênicos. Rev Bras Psiquiatr. 2004;26(Supl 1):14-7.

44. Bahls FRC, Ingbermann YK. Desenvolvimento escolar e abuso de drogas na adolescência. Estud Psicol. 2005;22(4):395-402.

45. Nascimento EC, Justo JS. Vidas errantes e alcoolismo: uma questão social. Psicol Reflex Crit. 2000;13(3):529-38.

46. United States Department of Health and Human Services. Substance Abuse and Mental Health Services Administration. Office of Applied Studies. National Household Survey on Drug Abuse, 2000. United States; 2000.

47. Sadock BJ, Sadock VA. Transtornos relacionados a substâncias. In: Sadock BJ, Sadock VA. Compêndio de psiquiatria - ciência do comportamento e psiquiatria clínica. Porto Alegre: Artmed; 2007.

48. Neri MC. Novo mapa das religiões. Rio de Janeiro: CPS/FGV; 2011.

49. Trigo A. Estado laico, acolhimento religioso: as discrepâncias no atendimento aos dependentes químicos em São Paulo. Último Andar. 2015;26:35-47.
50. Marlatt GA, Donovann DM. Prevenção de recaída - estratégias de manutenção no tratamento de comportamentos adictivos. 2a ed. Porto Alegre: Artmed; 2009.

51. Zenelatto N. Prevenção de recaída. In: Diehl A, Cordeiro DC, Laranjeira R, editores. Dependência química. Porto Alegre: Artmed; 2011.

52. Canavez MF, Alves AR, Canavez LS. Fatores predisponentes para 0 uso precoce de drogas por adolescentes. Cadernos UniFOA. 2010;14:57-63.

53. Figlie N, Fontes A, Moraes E, Payã R. Filhos de dependentes químicos com fatores de risco bio-psicossociais: necessitam de um olhar especial?. Rev Psiq Clín. 2004;31(2):53-62.

54. Paduani GF, Barbosa GA, Morais JCR, Pereira JCP, Almeida MF, Prado MM, et al. Consumo de álcool e fumo entre os estudantes da Faculdade de Medicina da Universidade Federal de Uberlândia. Rev Bras Educ Med. 2008;32(1):66-74.

55. Ribeiro M, Dunn J, Sesso R, Dias AC, Laranjeira R. Causes of death among crack cocaine users. Rev Bras Psiquiatr. 2006;28(3):196-202.

56. ergusson DM, Boden JM, Horwood LJ. Cannabis use and other illicit drug use: testing the cannabis gateway hypothesis. Addiction. 2006;101(4):556-69.

57. Sanchez ZM, Nappo SA. Sequência de drogas consumidas por usuários de crack e fatores interferentes. Rev Saúde Pública. 2002;36(4):420-30.

58. Boden ZM, Fergusson DM. Alcohol and depression. Addiction. 2011;106(5):906-14.

59. Ribeiro M, Laranjeira R, Cividanes G. Transtorno bipolar do humor e uso indevido de substâncias psicoativas. Rev Psiquiatr Clín. 2005;32(Supl 1):78-88.

60. American Psychiatry Association. Diagnostic and Statistical Manual of Mental disorders DSM-5. 5th ed. Washington: American Psychiatric Association; 2013.

61. Merikangas KR, Mehta RL, Molnar BE, Walters EE, Swendsen JD, Aguilar-Gaziola S, et al. Comorbidity of substance use disorders with mood and anxiety disorders: results of the International Consortium in Psychiatric Epidemiology. Addict Behav. 1998;23(6):893907.

62. Biederman J, Wilens T, MickE, Milberger S, Spencer TJ, Faraone SV. Psychoactive substance use disorders in adults with attention deficit hyperactivity disorder (ADHD): effects of ADHD and psychiatric comorbidity. Am J Psychiatry. 1995;152(11):1652-8.

63. Santos EG, Siqueira MM. Prevalência dos transtornos mentais na populaçãa adulta brasileira: uma revisão sistemática de 1997 a 2009. J Bras Psiquiatr. 2010;59(3):238-46. 
Anexo 1. Ficha de dados sociodemográficos/antecedentes pessoais e familiares

1. Nome:

2. Data de nascimento: 3. Idade:

4. Procedência:

5. Estado civil:

( ) Solteiro( ) Casado( ) Divorciado( ) Separado ( ) Viúvo( ) Uniãoconsensual

6. Prole:

( ) Não ( ) Sim $\rightarrow$ Quantos:

7. Escolaridade:

( ) Nunca estudou ( ) Fundamental incompleto ( ) Fundamental completo

( ) Médio incompleto ( ) Médio completo ( ) Superior incompleto

( ) Superior completo ( ) Curso técnico incompleto ( ) Curso técnico completo

8. Religião:

( ) Católico( ) Evangélico( ) Espírita ( ) Ateu ( ) Outras

9. Profissão:

( ) Empregado $\rightarrow$ Profissão atual:

( ) Desempregado ( ) Outros

10. Renda pessoal:

( ) < ou = 1 salário mínimo ( ) > que 1 salário mínimo, até 2 salários mínimos

( ) ) 2 salários mínimos, até 3 salários mínimos ( ) > 3 salários mínimos

Obs.: Maior responsável pelo sustento da família:

11. Dependência química:

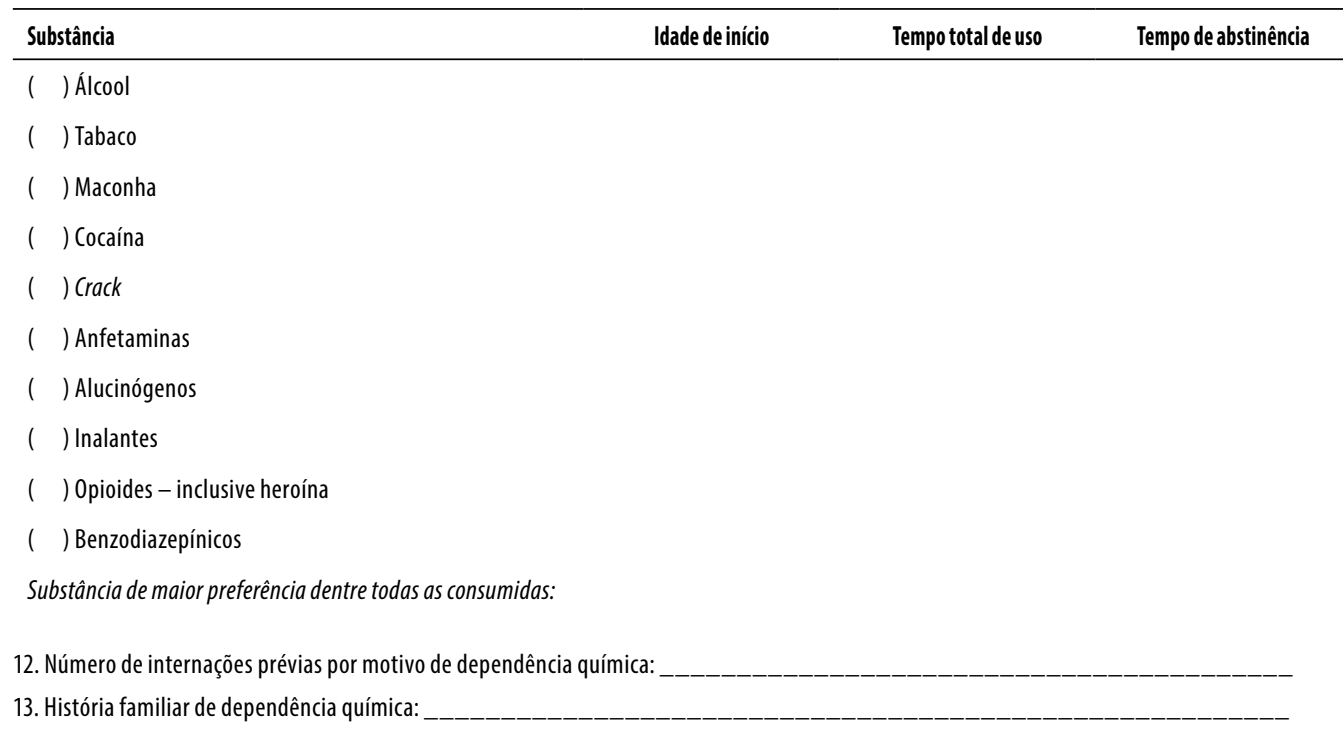

14. Comorbidades clínicas principais: 